\title{
OPERADOR DIPOLO-DIPOLO NA BASE DE MOMENTO ANGULAR: UM COMPLEMENTO AO ESTUDO DE RESSONÂNCIA MAGNÉTICA NUCLEAR
}

\author{
Rita de Cássia de Oliveira Sebastião e João Pedro Braga* \\ Departamento de Química, Instituto de Ciências Exatas, Universidade Federal de Minas Gerais, CP702, 31270-901 Belo Horizonte \\ - MG, Brasil
}

Recebido em 3/4/07; aceito em 11/10/07; publicado na web em 2/4/08

\begin{abstract}
DIPOLE-DIPOLE OPERATOR IN ANGULAR MOMENTUM BASIS: A COMPLEMENTARY STUDY IN NUCLEAR MAGNETIC RESONANCE. The relationship between the magnetic dipole-dipole potential energy function and its quantum analogue is presented in this work. It is assumed the reader is familiar with the classical expression of the dipolar interaction and has basic knowledge of the quantum mechanics of angular momentum. Except for these two points only elementary steps are involved.
\end{abstract}

Keywords: dipole interaction; angular momentum; NMR

\section{INTRODUÇÃO}

\section{Motivação}

O presente artigo teve como objetivo principal ser um complemento ao estudo da ressonância magnética nuclear. A energia potencial gerada pela interação entre um dipolo magnético com o campo magnético criado por um segundo dipolo magnético, interação dipolodipolo, transforma-se numa forma bastante útil para interpretação de efeitos quânticos presentes na ressonância magnética de sólidos. A ligação entre essas duas formas, uma pertencente ao eletro-magnetismo clássico e a outra à mecânica quântica, não é óbvia e as passagens envolvidas são, invariavelmente, abandonadas em livros e artigos de ressonância magnética nuclear.

Por exemplo, o tratado de Abragam ${ }^{1}$ somente enuncia o resultado final. Outros seguem o mesmo caminho, como por exemplo nas referências 2, 3 e 4 . Alguns autores, como por exemplo na referência 5 , não fornecem a bibliografia em que tal prova possa ser encontrada e outros a associam como uma alphabetic soup ${ }^{4}$ pois, normalmente, são usadas letras de $A$ a $F$ para se obter a expressão final. Alguns autores citam o artigo de van Vleck $^{6}$ como referência desse Hamiltoniano. Nesse artigo a dedução do Hamiltoniano não é apresentada.

Entretanto, como o leitor irá perceber, existe uma razão para que essa prova não apareça em livros textos e artigos específicos. A prova é demasiadamente longa para um artigo ou para um livro texto no assunto. Ter-se-ia, no caso de livros, de se ter um capítulo específico para essa prova. Contudo, o aluno que começa a sua leitura, querendo entender as passagens envolvidas, fica com a impressão de que algo está faltando no estudo dos princípios básicos da ressonância magnética nuclear. Obviamente que esse Hamiltoniano foi deduzido, mas não é apresentado nos livros e muito menos o caminho para a sua prova. Pretendeu-se, neste artigo, preencher essa lacuna no ensino de ressonância magnética nuclear.

Uma outra maneira da dedução desse Hamiltoniano é também possível, mas requer o conhecimento de tensores, coeficientes de Clebsch-Gordon e o teorema de Wigner-Eckart. Esse tipo de prova é discutida, por exemplo, na referência 7. O Hamiltoniano obtido por esse caminho esconde uma forma útil e passagens extras de-

*e-mail: jpbraga@ufmg.br vem ser feitas.

$\mathrm{O}$ presente artigo começa apresentando o pré-requisito para acompanhar a dedução apresentada. O leitor deve ter conhecimento da energia potencial entre dois dipolos ${ }^{8}$ e de combinação de operadores de momentos angulares. ${ }^{9}$ Somente passagens elementares são requeridas durante o desenvolvimento do artigo.

\section{Base teórica}

A energia potencial, $E_{\mathrm{p}}$, gerada pela interação entre um dipolo magnético, $\vec{m}_{1}$ e o campo magnético, $\vec{B}_{2}$, criado por um segundo dipolo, $\vec{m}_{2}$, é igual a $E_{p}=-\vec{m}_{1} \cdot \vec{B}_{2}$, podendo ser desenvolvida na forma, ${ }^{8}$

$$
E_{p}=-\frac{\mu_{0}}{4 \pi}\left(\frac{3\left(\vec{m}_{1} \cdot \vec{R}\right)\left(\vec{m}_{2} \cdot \vec{R}\right)}{R^{5}}-\frac{\vec{m}_{1} \cdot \vec{m}_{2}}{R^{3}}\right)
$$

Usando a relação entre momento magnético e spin nuclear, $\vec{I}$, desenvolve-se,

$E_{p}=-\frac{\mu_{0}}{4 \pi} \gamma_{1} \gamma_{2}\left(\frac{3\left(\vec{I}_{1} \cdot \vec{R}\right)\left(\vec{I}_{2} \cdot \vec{R}\right)}{R^{5}}-\frac{\vec{I}_{1} \cdot \vec{I}_{2}}{R^{3}}\right)$

em que $\gamma_{1}$ e $\gamma_{2}$ são as razões giro-magnéticas dos núcleos em consideração. Essa energia potencial pode ser colocada na forma de operador,

$$
\hat{H}=-\frac{\mu_{0}}{4 \pi} \gamma_{1} \gamma_{2}\left(\frac{3\left(\hat{I}_{1} \cdot \hat{R}\right)\left(\hat{I}_{2} \cdot \hat{R}\right)}{R^{5}}-\frac{\hat{I}_{1} \cdot \hat{I}_{2}}{R^{3}}\right)
$$

Ainda uma expressão mais útil deve ser obtida. Pela definição do vetor unitário na direção radial, $\hat{n}=\frac{\hat{R}}{R}$, transforma-se o operador em

$$
\hat{H}=-\frac{\mu_{0}}{4 \pi R^{3}} \gamma_{1} \gamma_{2}\left(3\left(\hat{I}_{1} \cdot \hat{n}\right)\left(\hat{I}_{2} \cdot \hat{n}\right)-\hat{I}_{1} \cdot \hat{I}_{2}\right)
$$




\section{Momento angular}

Assume-se, também, que o leitor tenha conhecimento de álgebra elementar de momento angular, por exemplo, ter conhecimento das relações: ${ }^{9}$

$$
\hat{J}_{ \pm}=\hat{J}_{x} \pm i \hat{J}_{y}
$$

e, por conseguinte,

$\hat{J}_{x}=\frac{\hat{J}_{+}+\hat{J}_{-}}{2}$

$\hat{J}_{y}=\frac{\hat{J}_{+}-\hat{J}_{-}}{2 i}$

em que $\hat{J}$ representa o momento angular.

A forma que os operadores criação e aniquilação, respectivamente, $\hat{J}$ e $\hat{J}$, atuam na função não é um pré-requisito para se entender o presente trabalho, mas é útil na interpretação do Hamiltoniano final.

\section{Coordenadas esféricas}

Para provar a expressão desejada usam-se coordenadas esféricas para orientar um momento de dipolo em relação ao outro. Um dos momentos de dipolo terá coordenadas angulares $\left(\theta_{1}, \phi_{1}\right)$ e o outro, coordenadas $\left(\theta_{2}, \phi_{2}\right)$. A orientação de um dipolo em relação ao outro terá coordenadas $(\theta, \phi)$. A situação é análoga ao problema de dois elétrons, no cálculo da integral de Coulomb, ${ }^{9}$ Portanto, podese escrever, $\hat{n}=\operatorname{sen} \theta \cos \phi \vec{i}+\operatorname{sen} \theta \operatorname{sen} \phi \vec{j}+\cos \theta \vec{k}$.

Também

$\hat{I}_{1}=\hat{I}_{1 x} \vec{i}+\hat{I}_{1 y} \vec{j}+\hat{I}_{1 z} \vec{k}$

$\hat{I}_{2}=\hat{I}_{2 x} \vec{i}+\hat{I}_{2 y} \vec{j}+\hat{I}_{2 z} \vec{k}$

Para simplificar a notação, o símbolo de operador será omitido do operador momento angular. Definindo, $C=-\frac{\mu_{0}}{4 \pi R^{3}} \gamma_{1} \gamma_{2}$ e $\hat{H}^{\prime}=\frac{\hat{H}}{C}$, desenvolve-se

$\hat{H}^{\prime}=3\left(I_{1 x} \operatorname{sen} \theta \cos \phi+I_{1 y} \operatorname{sen} \theta \operatorname{sen} \phi+I_{1 z} \cos \theta\right)$

$\times\left(I_{2 x} \operatorname{sen} \theta \cos \phi+I_{2 y} \operatorname{sen} \theta \operatorname{sen} \phi+I_{2 z} \cos \theta\right)$

$-I_{1 x} I_{2 x}+I_{1 y} I_{2 y}+I_{1 z} I_{2 z}$

$=3 A^{\prime}-\left(I_{1 x} I_{2 x}+I_{1 y} I_{2 y}+I_{1 z} I_{2 z}\right)$

Desenvolvendo o produto, termo $A^{\prime}$

$A^{\prime}=I_{1 x} I_{2 x} \operatorname{sen}^{2} \theta \cos ^{2} \phi+I_{1 x} I_{2 y} \operatorname{sen}^{2} \theta \operatorname{sen} \phi \cos \phi$

$+I_{1 x} I_{2 z} \operatorname{sen} \theta \cos \theta \cos \phi+I_{1 y} I_{2 x} \operatorname{sen}^{2} \theta \operatorname{sen} \phi \cos \phi$

$+I_{1 y} I_{2 y} \operatorname{sen}^{2} \theta \operatorname{sen}^{2} \phi+I_{1 x} I_{2 z} \operatorname{sen} \theta \cos \theta \operatorname{sen} \phi$

$+I_{1 z} I_{2 x} \operatorname{sen} \theta \cos \theta \operatorname{sen} \phi+I_{1 z} I_{2 y} \operatorname{sen} \theta \cos \theta \cos \phi$

$+I_{1 z} I_{2 z} \cos ^{2} \theta$

Esse termo deverá ser substituído no operador $\hat{\mathrm{H}}$ ', para fornecer
$\hat{H}^{\prime}=I_{1 z} I_{2 z}\left(3 \cos ^{2} \theta-1\right)+I_{1 x} I_{2 x}\left(3 \operatorname{sen}^{2} \theta \cos ^{2} \phi-1\right)$

$+I_{1 y} I_{2 y}\left(3 \operatorname{sen}^{2} \theta \operatorname{sen}^{2} \phi-1\right)+3 I_{1 x} I_{2 y}+I_{1 y} I_{2 x}\left(\operatorname{sen}^{2} \theta \operatorname{sen} \phi \cos \phi\right)$

$+3\left(I_{1 x} I_{2 z}+I_{1 z} I_{2 x}\right) \operatorname{sen} \theta \cos \theta \cos \phi$

$+3\left(I_{1 y} I_{2 z}+I_{1 z} I_{2 y}\right) \operatorname{sen} \theta \cos \theta \operatorname{sen} \phi$

$-\left(I_{1 x} I_{2 x}+I_{1 y} I_{2 y}+I_{1 z} I_{2 z}\right)$

\section{Operador criação e aniquilação}

Para se chegar ao resultado desejado, os operadores $I_{x}, I_{y}$ para os dipolos 1 e 2 devem ser substituídos pelas relações (6). Para o termo $I_{1 x}, I_{2 x}$ desenvolve-se

$$
\begin{aligned}
& I_{1 x} I_{2 x}=\frac{I_{1+}+I_{1-}}{2} \times \frac{I_{2+}+I_{2-}}{2} \\
& =\frac{1}{4}\left(I_{1+} I_{2+}+I_{1+} I_{2-}+I_{1-} I_{2+}+I_{1-} I_{2-}\right)
\end{aligned}
$$

As outras expressões são obtidas de forma análoga. Todas essas relações podem ser resumidas na forma

$$
\begin{aligned}
& I_{1 x} I_{2 x}=\frac{1}{4}\left(I_{1+} I_{2+}+I_{1+} I_{2-}+I_{1-} I_{2+}+I_{1-} I_{2-}\right) \\
& I_{1 x} I_{2 y}=\frac{1}{4 i}\left(I_{1+} I_{2+}-I_{1+} I_{2-}+I_{1-} I_{2+}-I_{1-} I_{2-}\right) \\
& I_{1 z} I_{2 x}=\frac{1}{2}\left(I_{1 z} I_{2+}+I_{1 z} I_{2-}\right) \\
& I_{1 z} I_{2 y}=\frac{1}{2 i}\left(I_{1 z} I_{2+}-I_{1 z} I_{2-}\right) \\
& I_{1 x} I_{2 z}=\frac{1}{2}\left(I_{1+} I_{2 z}+I_{1-} I_{2 z}\right) \\
& I_{1 y} I_{2 x}=\frac{1}{4 i}\left(I_{1+} I_{2+}+I_{1+} I_{2-}-I_{1-} I_{2+}-I_{1-} I_{2-}\right) \\
& I_{1 y} I_{2 y}=-\frac{1}{4}\left(I_{1+} I_{2+}-I_{1+} I_{2-}-I_{1-} I_{2+}+I_{1-} I_{2-}\right) \\
& I_{1 y} I_{2 z}=\frac{1}{2 i}\left(I_{1+} I_{2 z}-I_{1-} I_{2 z}\right)
\end{aligned}
$$

A substituição das expressões (9) e (12) no Hamiltoniano H', Equação (8), resultará em

$$
\begin{aligned}
& \hat{H}^{\prime}=I_{1 z} I_{2 z}\left(3 \cos ^{2} \theta-1\right) \\
& +\frac{1}{4}\left(I_{1+} I_{2+}+I_{1+} I_{2-}+I_{1-} I_{2+}+I_{1-} I_{2-}\right)\left(3 \operatorname{sen}^{2} \theta \cos ^{2} \phi-1\right) \\
& -\frac{1}{4}\left(I_{1+} I_{2+}-I_{1+} I_{2-}-I_{1-} I_{2+}+I_{1-} I_{2-}\right)\left(3 \operatorname{sen}^{2} \theta \operatorname{sen}^{2} \phi-1\right) \\
& +3 \times\left\{\frac{1}{4 i}\left(I_{1+} I_{2+}-I_{1+} I_{2-}+I_{1-} I_{2+}-I_{1-} I_{2-}\right)\right. \\
& \left.+\frac{1}{4 i}\left(I_{1+} I_{2+}+I_{1+} I_{2-}-I_{1-} I_{2+}-I_{1-} I_{2-}\right)\right\}\left(\operatorname{sen}^{2} \theta \operatorname{sen} \phi \cos \phi\right) \\
& +3\left(\frac{1}{2}\left(I_{1+} I_{2 z}+I_{1-} I_{2 z}\right)+\frac{1}{2}\left(I_{1 z} I_{2+}+I_{1 z} I_{2-}\right)\right) \operatorname{sen} \theta \cos \theta \cos \phi \\
& +3\left(\frac{1}{2 i}\left(I_{1+} I_{2 z}-I_{1-} I_{2 z}\right)+\frac{1}{2 i}\left(I_{1 z} I_{2+}-I_{1 z} I_{2-}\right)\right) \operatorname{sen} \theta \cos \theta \operatorname{sen} \phi
\end{aligned}
$$




\section{Termos semelhantes}

Os termos semelhantes devem ser agora agrupados. Por exemplo, para os termos que contêm $1 / 4 I_{1+} I_{2}$

$C^{\prime}=\frac{1}{4} I_{1+} I_{2-}\left(3 \operatorname{sen}^{2} \theta \cos ^{2} \phi-1+3 \operatorname{sen}^{2} \theta \cos ^{2} \phi-1\right)$

$-\frac{3}{i} \operatorname{sen}^{2} \theta \operatorname{sen} \phi \cos \phi+\frac{3}{i} \operatorname{sen}^{2} \theta \operatorname{sen} \phi \cos \phi$

$=\frac{1}{4} I_{1+} I_{2-}\left(3 \operatorname{sen}^{2} \theta-2\right)$

$=\frac{1}{4} I_{1+} I_{2-}\left(3\left(1-\cos ^{2} \theta-2\right)\right.$

$=\frac{1}{4} I_{1+} I_{2-}\left(3 \cos ^{2} \theta-1\right)$

De maneira semelhante desenvolve-se para $1 / 4 I_{1-} I_{2+}$

$D^{\prime}=\frac{1}{4} I_{1-} I_{2+}\left(3 \operatorname{sen}^{2} \theta \cos ^{2} \phi-1+3 \operatorname{sen}^{2} \theta \cos ^{2} \phi-1\right)$

$-\frac{3}{i} \operatorname{sen}^{2} \theta \operatorname{sen} \phi \cos \phi+\frac{3}{i} \operatorname{sen}^{2} \theta \operatorname{sen} \phi \cos \phi$

$=\frac{1}{4} I_{1-} I_{2+}\left(3 \operatorname{sen}^{2} \theta-2\right)$

$=\frac{1}{4} I_{1-} I_{2+}\left(3\left(1-\cos ^{2} \theta-2\right)\right.$

$=\frac{1}{4} I_{1-} I_{2+}\left(3 \cos ^{2} \theta-1\right)$

Portanto, o Hamiltoniano parcial assume a forma

$\hat{H}^{\prime}=I_{1 z} I_{2 z}\left(3 \cos ^{2} \theta-1\right)$

$-\frac{1}{4}\left(I_{1+} I_{2-}+I_{1-} I_{2+}\right)\left(3 \cos ^{2} \theta-1\right)=\left(C^{\prime}+D^{\prime}\right)$

$+\frac{1}{4}\left(I_{1+} I_{2+}+I_{1-} I_{2-}\right)\left(3 \operatorname{sen}^{2} \theta \cos ^{2} \phi-1\right)$

$-\frac{1}{4}\left(I_{1+} I_{2+}-I_{1-} I_{2-}\right)\left(3 \operatorname{sen}^{2} \theta \operatorname{sen}^{2} \phi-1\right)$

$+3 \times\left\{\frac{1}{4 i}\left(I_{1+} I_{2+}-I_{1-} I_{2-}\right)\right.$

$\left.+\frac{1}{4 i}\left(I_{1+} I_{2+}-I_{1-} I_{2-}\right)\right\}\left(\operatorname{sen}^{2} \theta \operatorname{sen} \phi \cos \phi\right)$

$+3\left(\frac{1}{2}\left(I_{1+} I_{2 z}+I_{1-} I_{2 z}\right)+\frac{1}{2}\left(I_{1 z} I_{2+}+I_{1 z} I_{2-}\right)\right) \operatorname{sen} \theta \cos \theta \cos \phi$

$+3\left(\frac{1}{2 i}\left(I_{1+} I_{2 z}-I_{1-} I_{2 z}\right)+\frac{1}{2 i}\left(I_{1 z} I_{2+}-I_{1 z} I_{2-}\right)\right) \operatorname{sen} \theta \cos \theta \operatorname{sen} \phi$

Os termos em que $I_{1+} I_{2 z}$ e $I_{1 \mathrm{z}} I_{2+}$ estão presentes reagrupam-se na forma

$E^{\prime}=\frac{3}{2} I_{1+} I_{2 z} \operatorname{sen} \theta \cos \theta\left(\cos \phi+\frac{1}{i} \operatorname{sen} \phi\right)$

$+\frac{3}{2} I_{1 z} I_{2+} \operatorname{sen} \theta \cos \theta\left(\cos \phi+\frac{1}{i} \operatorname{sen} \phi\right)$

$=\frac{3}{2}\left(I_{1+} I_{2 z}+I_{1 z} I_{2+}\right) \operatorname{sen} \theta \cos \theta \exp (-i \phi)$

e pelo mesmo raciocínio, para $I_{1-} I_{2 \mathrm{z}}$ e $I_{1 \mathrm{z}} I_{2}$

$F^{\prime}=\frac{3}{2} I_{1-} I_{2 z} \operatorname{sen} \theta \cos \theta\left(\cos \phi-\frac{1}{i} \operatorname{sen} \phi\right)$

$+\frac{3}{2} I_{1 z} I_{2-} \operatorname{sen} \theta \cos \theta\left(\cos \phi-\frac{1}{i} \operatorname{sen} \phi\right)$

$=\frac{3}{2}\left(I_{1-} I_{2 z}+I_{1 z} I_{2-}\right) \operatorname{sen} \theta \cos \theta \exp (i \phi)$
A substituição de $E^{\prime}$ e $F^{\prime}$ fornece um Hamiltoniano parcial

$\hat{H}^{\prime}=I_{1 z} I_{2 z}\left(3 \cos ^{2} \theta-1\right)$

$-\frac{1}{4}\left(I_{1+} I_{2-}+I_{1-} I_{2+}\right)\left(3 \cos ^{2} \theta-1\right)=\left(C^{\prime}+D^{\prime}\right)$

$+\frac{3}{2}\left(I_{1+} I_{2 z}+I_{1 z} I_{2+}\right) \operatorname{sen} \theta \cos \theta \exp (-i \phi)=\left(E^{\prime}\right)$

$+\frac{3}{2}\left(I_{1-} I_{2 z}+I_{1 z} I_{2-}\right) \operatorname{sen} \theta \cos \theta \exp (i \phi)=\left(F^{\prime}\right)$

$+\frac{1}{4}\left(I_{1+} I_{2+}+I_{1-} I_{2-}\right)\left(3 \operatorname{sen}^{2} \theta \cos ^{2} \phi-1\right)$

$-\frac{1}{4}\left(I_{1+} I_{2+}-I_{1-} I_{2-}\right)\left(3 \operatorname{sen}^{2} \theta \operatorname{sen}^{2} \phi-1\right)$

$+3 \times\left\{\frac{1}{4 i}\left(I_{1+} I_{2+}-I_{1-} I_{2-}\right)\right.$

$\left.+\frac{1}{4 i}\left(I_{1+} I_{2+}-I_{1-} I_{2-}\right)\right\}\left(\operatorname{sen}^{2} \theta \operatorname{sen} \phi \cos \phi\right)$

O raciocínio deve ser continuado, restando apenas dois tipos de termos. Por exemplo o termo $I_{1+} I_{2+}$ aparece em quatro lugares e pode ser reorganizado na forma

$G^{\prime}=\frac{1}{4} I_{1+} I_{2+}\left\{3 \operatorname{sen}^{2} \theta \cos ^{2} \phi-1-3 \operatorname{sen}^{2} \theta \operatorname{sen}^{2} \phi+1\right.$

$\left.+\frac{3}{i} \cdot 2\left(\operatorname{sen}^{2} \theta \operatorname{sen} \phi \cos \phi\right)\right\}$

$=\frac{1}{4} I_{1+} I_{2+}\left\{3 \operatorname{sen}^{2} \theta\left(\frac{1+\cos 2 \phi}{2}\right)-3 \operatorname{sen}^{2} \theta\left(\frac{1-\cos 2 \phi}{2}\right)\right.$

$+\frac{3}{i} \cdot 2\left(\operatorname{sen}^{2} \theta \operatorname{sen} \phi \cos \phi\right)$

$=\frac{1}{4} I_{1+} I_{2+}\left(3 \operatorname{sen}^{2} \theta \cos 2 \phi+\frac{3}{i} \operatorname{sen}^{2} \theta \operatorname{sen} 2 \phi\right)$

$=\frac{3}{4} I_{1+} I_{2+} \operatorname{sen}^{2} \theta(\cos 2 \phi-i \operatorname{sen} 2 \phi)$

$=\frac{3}{4} I_{1+} I_{2+} \operatorname{sen}^{2} \theta \exp (-2 i \phi)$

Observe que as relações trigonométricas, $\cos ^{2} x=\frac{1+\cos 2 x}{2}$, $\operatorname{sen}^{2} x=\frac{1-\cos 2 x}{2}$ e $\exp (i x)=\cos x+i$ sen $x$ foram empregadas. Para $I_{1-2} I_{2-}$ (note a semelhança com a dedução do termo $G^{\prime}$ ) desenvolve-se $I^{\prime}=\frac{1}{4} I_{1-} I_{2-}\left\{\operatorname{sen}^{2} \theta \cos ^{2} \phi-1-3 \operatorname{sen}^{2} \theta \operatorname{sen}^{2} \phi+1\right.$ $\left.-\frac{3}{i} \cdot 2\left(\operatorname{sen}^{2} \theta \operatorname{sen} \phi \cos \phi\right)\right\}$

$=\frac{1}{4} I_{1-} I_{2-}\left\{3 \operatorname{sen}^{2} \theta\left(\frac{1+\cos 2 \phi}{2}\right)-3 \operatorname{sen}^{2} \theta\left(\frac{1-\cos 2 \phi}{2}\right)\right.$

$\left.-\frac{3}{i} \cdot 2\left(\operatorname{sen}^{2} \theta \operatorname{sen} \phi \cos \phi\right)\right\}$

$=\frac{1}{4} I_{1-} I_{2-}\left(3 \operatorname{sen}^{2} \theta \cos 2 \phi-\frac{3}{i} \operatorname{sen}^{2} \theta \operatorname{sen} 2 \phi\right)$

$=\frac{3}{4} I_{1-} I_{2-} \operatorname{sen}^{2} \theta(\cos 2 \phi+i \operatorname{sen} 2 \phi)$

$=\frac{3}{4} I_{1-} I_{2-} \operatorname{sen}^{2} \theta \exp (2 i \phi)$ 
$\hat{H}^{\prime}=\frac{1}{4} I_{1 z} I_{2 z}\left(3 \cos ^{2} \phi-1\right)$

$-\frac{1}{4}\left(I_{1+} I_{2-}+I_{1-} I_{2+}\right)\left(3 \cos ^{2} \phi-1\right)=\left(C^{\prime}+D^{\prime}\right)$

$+\frac{3}{2}\left(I_{1+} I_{2 z}+I_{1 z} I_{2+}\right) \operatorname{sen} \theta \cos \theta \exp (-i \phi)=\left(E^{\prime}\right)$

$+\frac{3}{2}\left(I_{1-} I_{2 z}+I_{1 z} I_{2-}\right) \operatorname{sen} \theta \cos \theta \exp (i \phi)=\left(F^{\prime}\right)$

$+\frac{3}{4} I_{1+} I_{2+} \operatorname{sen}^{2} \theta \exp (-2 i \phi)=\left(G^{\prime}\right)$

$+\frac{3}{4} I_{1-} I_{2-} \operatorname{sen}^{2} \theta \exp (2 i \phi)=\left(I^{\prime}\right)$

Retornando com as definições usadas

$\hat{H}=-\frac{\mu_{0}}{4 \pi} \gamma_{1} \gamma_{2}\left\{\hat{I}_{1 z} \hat{I}_{2 z}\left(3 \cos ^{2} \phi-1\right)\right.$

$-\frac{1}{4}\left(\hat{I}_{1+} \hat{I}_{2-}+\hat{I}_{1-} \hat{I}_{2+}\right)\left(3 \cos ^{2} \phi-1\right)$

$+\frac{3}{2}\left(\hat{I}_{1+} \hat{I}_{2 z}+\hat{I}_{1 z} \hat{I}_{2+}\right) \operatorname{sen} \theta \cos \theta \exp (-i \phi)$

$+\frac{3}{2}\left(\hat{I}_{1-} \hat{I}_{2 z}+\hat{I}_{1 z} \hat{I}_{2-}\right) \operatorname{sen} \theta \cos \theta \exp (i \phi)$

$+\frac{3}{4} \hat{I}_{1+} \hat{I}_{2+} \operatorname{sen}^{2} \theta \exp (-2 i \phi)$

$+\frac{3}{4} \hat{I}_{1-} \hat{I}_{2-} \operatorname{sen}^{2} \theta \exp (2 i \phi)$

que é o resultado desejado. Como enfatizado, somente passagens elementares foram necessárias para se estabelecer o operador na forma final. A relação entre a quantidade clássica, $E_{p}=-\vec{m}_{1} \cdot \vec{B}_{2}$, e seu correspondente quântico, Equação 23, fica, portanto, estabelecida.

\section{CONCLUSÃO}

Ao contrário da prova do Hamiltoniano (Equação 23), as im- plicações desse Hamiltoniano na base de momento angular são discutidas com clareza nas referências citadas. Como exemplo, uma aplicação elegante do operador dipolo-dipolo é na simulação de espectros de alta resolução de moléculas pequenas parcialmente orientadas em cristais líquidos. O primeiro e segundo termos acoplam estados com $\Delta m=0$, o terceiro e quarto com $\Delta m= \pm 1$ e o quinto e sexto termos com $\Delta m= \pm 2$, em que $m$ é a soma dos autovalores do momento angular de spin. A interação dipolar pode ser entendida como uma pequena perturbação na interação Zeeman em que todos os termos na Equação 23, exceto os dois primeiros, apresentam contribuições desprezíveis. O conhecimento da representação dos operadores, $I_{z}, I_{+}$e I permite que o Hamiltoniano na forma (23) seja representado numa base adequada. Efeitos no espectro da ressonância magnética ${ }^{1}$ ou o estudo da dinâmica de spin, como na referência 10, podem ser, por conseguinte, estudados.

\section{AGRADECIMENTOS}

Ao CNPq e à FAPEMIG pelo apoio financeiro.

\section{REFERÊNCIAS}

1. Abragam, A.; Principles of Nuclear Magnetism, Oxford University Press: Oxford, 1983.

2. Ernst, R. R.; Bodenhausen, G.; Wokaun, A.; Principles of nuclear magnetic resonance in one and two dimensions, Oxford University Press: Oxford, 2003.

3. Slichter, C. P.; Principles of Magnetic Resonance, Springer Verlag: New York, 2006.

4. Gerstein, B. C.; Dybowski, C. R.; Transient Techniques in NMR of Solids, Academic Press: London, 1985.

5. Becker, E. D.; High Resolution NMR: Theory and Chemical Applications, Academic Press: London, 1999.

6. van Vleck, J. H.; Phys. Rev. 1948, 74, 1168.

7. Rose, M. E.; Elementary Theory of Angular Momentum, Dover: New York, 1995.

8. Griffiths, D. J.; Introduction to Electrodynamics, Benjamin Cummings: New York, 1998.

9. Braga, J. P.; Fundamentos de Química Quântica, Editora UFV: Viçosa, 2007.

10. Solomon, I.; Phys. Rev. 1955, 99 , 559. 